\title{
Dendrimers and their Applications as Novel Drug Delivery Carriers
}

\author{
Surendra Tripathy ${ }^{1} *$ and Malay K Das ${ }^{2}$ \\ ${ }^{1}$ Division of Pharmaceutics, Varanasi College of Pharmacy, Varanasi-221006, India. \\ ${ }^{2}$ Department of Pharmaceutical Sciences, Dibrugarh University, Dibrugarh-786004, India.
}

\begin{tabular}{l} 
ARTICLE INFO \\
\hline Article history: \\
Received on: $24 / 07 / 2013$ \\
Revised on: $18 / 08 / 2013$ \\
Accepted on: 03/09/2013 \\
Available online: $30 / 09 / 2013$ \\
\hline Key words: \\
Dendrimers, PAMAM, \\
monodispersity, nanocarriers, \\
targeted, PEGylation.
\end{tabular}

\begin{abstract}
Dendrimers are novel synthetic polymeric systems having improved physical and chemical properties due to their unique three dimensional architecture. Dendrimers have a well defined size, shape, molecular weight and monodispersity. These are compatible with drug moieties as well as bioactive molecules like DNA, heparin and other polyanions. The nanoscopic size and recognition abilities make dendrimers as ideal building blocks for self-assembly and self-organization systems. The cavities inside the dendritic structure can be modified to incorporate hydrophobic and hydrophilic drugs. The terminal groups are modified to attach antibodies and bioactive substances for targeting purpose along with providing miscibility, reactivity and solubility. Currently, dendrimers are of great interest for delivering drug molecules via different routes as a nanocarrier. Toxicity problems associated with cationic dendrimers are overcome by PEGylation, which neutralizes the charge on them. Dendrimers possess suitable properties to establish itself as a potential carrier for delivery of therapeutic agents irrespective of certain synthetic and regulatory constraints. This review contains various structural aspects and properties of dendrimers along with their pharmaceutical application as a potential novel drug delivery carrier.
\end{abstract}

\section{INTRODUCTION}

Dendrimers are a new class of polymeric materials. A dendrimer is typically symmetrical around the core and often adopts a spherical three dimensional architecture that provides a high degree of surface functionality and versatility. The first successful attempt to create and design dendritic structures by organic synthesis was carried out by Vogtle et al. in 1978 (Buhleir et al., 1978). However after this in the early 1980 Donald Tomalia and his co-workers had worked in this field at Dow chemicals (Tomalia et al., 1985). The first synthesized dendrimers were polyamidoamines (PAMAM). They are also known as starbust dendrimers. The term starbust is a trademark of the Dow chemicals company. Ammonia was used as the core molecule. The term originates from "Dendron" meaning a tree in Greek. At the same time Newkome group independently reported

* Corresponding Author

Surendra Tripathy, Division of Pharmaceutics,

Varanasi College of Pharmacy, Babatpur, Varanasi-221006, Uttar

Pradesh, India. Phone: 0532-2584092 (O), Fax: 05322584092 synthesis of similar macromolecules (Newkome et al., 1985). They called 'arborols' (from latin word 'arbor') also meaning a tree.

The term "cascade molecule" is also used, but dendrimer is the best established one. One of the examples of dendritic structure is in the central nervous system and the brain, a large amount of cells growing into dendritic structures in order to gain the largest exchange of material and information with the surrounding tissue. A striking example of dendritic structures in nature discovered just recently is the tremendous number of foot hairs on gecko's feet.

These foot hairs called "setae", form impressive dendritic network of tiny foot hairs "spatula", enabling the gecko to stick to surfaces through dry adhesion without the need of humidity to create surface tension. The dendritic pattern enhances greatly the sum total function of the system due to the synergistic action of single entities carried on the system only due to the unique dendritic presentation of the system. 


\section{MOLECULAR STRUCTURE, DENDRIMER GENERATIONS AND ITS COMPONENTS}

Dendrimers are built from a starting atom, such as nitrogen, to which carbon and other elements are added by a repeating series of chemical reactions that produce a spherical branching structure. As the process repeats, successive layers are added and the sphere can be expanded to the desired size by the investigator. The final entity is spherical macromolecular structure whose size is similar to blood albumin and hemoglobin.

Dendrimers possess three separate architectural components (Pushkar et al., 2006, Sakthivel \& Florence, 2003), namely; (i) An initiator core (ii) Interior layers (generations) composed of repeating units, radically attached to the interior core (iii) Exterior (terminal functionality) attached to the outermost interior generations.

Dendrimer generation is the hyperbranching when going from the centre of the dendrimer towards the periphery, resulting in homostructural layers between the focal points (branching points). The number of focal points when going from the core towards the dendrimer surface is the generation number i.e a dendrimer having five focal points when going from the centre to the periphery is denoted as the 5th generation dendrimer. Here, this term is abbreviated to simply a G5-dendrimer, e.g. a 5th generation polypropylene imine is abbreviated to a "G5-PPI-" dendrimer. The core part of the dendrimer is sometimes denoted generation "zero", or in the terminology presented as "G0". The core structure thus presents no focal points, as hydrogen substituents are not considered focal points. Intermediates during the dendrimer synthesis are sometimes denoted half-generations (Pushkar et al., 2006, Zimmerman \& Lawless, 2001).

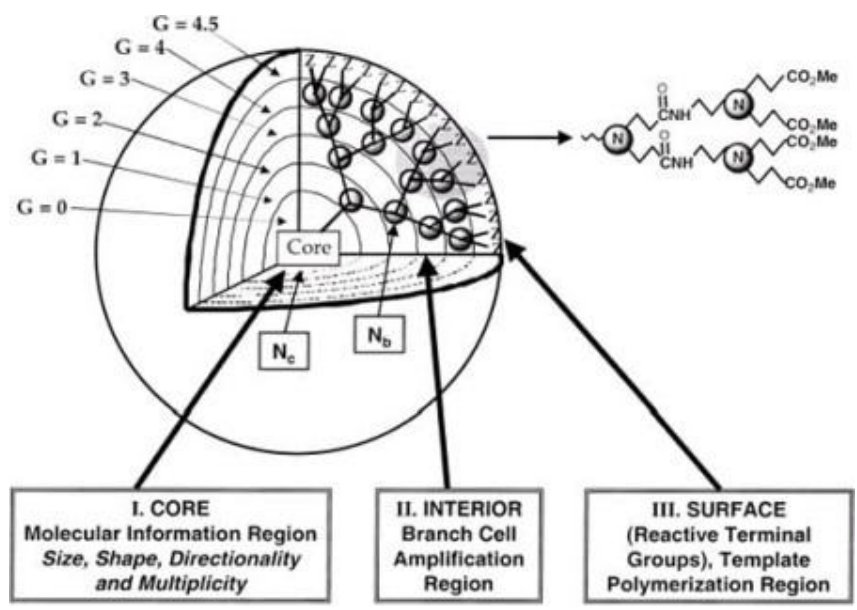

Fig. 1: Three dimensional projection of dendrimer core-shell architecture for $\mathrm{G}=4.5$ PAMAM dendrimer with principal architectural components (I) core, (II) interior \& (III) surface.

Dendrimers consist of three basic components: the shell, pincer and end group (Figure 1). The dendrimer shell is the homo-structural spatial segment between the focal points, the "generation space". The "outer shell" is the space between the last outer branching point and the surface. The "inner shells" are generally referred to as the dendrimer interior. In dendrimers, the outer shell consists of a varying number of pincers created by the last focal point before reaching the dendrimer surface.

In PPI and PAMAM dendrimers the number of pincers is half the number of surface groups (because in these dendrimers the chain divides into two chains in each focal point ). End group is also generally referred to as the "terminal group" or the "surface group" of the dendrimer. Dendrimers having amine end-groups are termed "aminoterminated dendrimers".

\section{TYPE OF DENDRIMERS}

\section{PAMAM Dendrimer}

Poly (amidoamine) dendrimers (PAMAM) are synthesized by the divergent method starting from ammonia or ethylenediamine initiator core reagents. PAMAM dendrimers are commercially available, usually as methanol solutions. Starburst dendrimers is applied as a trademark name for a sub-class of PAMAM dendrimers based on a trisaminoethylene-imine core. The name refers to the star like pattern observed when looking at the structure of the highgeneration dendrimers of this type in two-dimensions (Hawker \& Frechet, 1990).

\section{PAMAMOS Dendrimer}

Radially layered poly (amidoamine-organosilicon) dendrimers (PAMAMOS) are inverted unimolecular micelles that consist of hydrophilic, nucleophilic polyamidoamine (PAMAM) interiors and hydrophobic organosilicon (OS) exteriors. These dendrimers are exceptionally useful precursors for the preparation of honeycomb-like networks with nanoscopic PAMAM and OS domains. These are silicone containing first commercial dendrimers.

\section{PPI Dendrimer}

PPI-dendrimers stand for "Poly (Propylene Imine)" describing the propylamine spacer moieties in the oldest known dendrimer type developed initially by Vogtle. These dendrimers are generally poly-alkyl amines, having primary amines as end groups, the dendrimer interior consists of numerous of tertiary tris-propylene amines.

PPI dendrimers are commercially available up to G5, and has found widespread applications in the field of material science and biology. As an alternative name to PPI, POPAM is sometimes used to describe this class of dendrimers. POPAM stands for Poly (Propylene Amine), which closely resembles the PPI abbreviation. In addition, these dendrimers are also sometimes denoted "DAB-dendrimers" where DAB refers to the core structure, which is usually based on Diamino butane. 


\section{Tecto Dendrimer}

These are composed of a core dendrimer, surrounded by dendrimers of several steps to perform a function necessary for a smart therapeutic nanodevice. Different compounds perform varied functions ranging from diseased cell recognition, diagnosis of disease state, drug delivery, reporting outcomes of therapy.

\section{Multilingual Dendrimers}

In these dendrimers, the surface contains multiple copies of a particular functional group.

\section{Chiral Dendrimers}

The chirality in these dendrimers is based upon the construction of a constitutionally different but chemically similar branch to chiral core.

\section{Hybrid Dendrimers Linear Polymers}

These are hybrids (block or graft polymers) of dendritic and linear polymers and having properties of both.

\section{Amphiphilic Dendrimers}

They are built with two segregated sites of chain end, one half is electron donating and the other half is electron withdrawing.

\section{Micellar Dendrimers}

These are unimolecular micelles of water soluble hyper- branched polyphenylenes.

\section{Multiple Antigen Peptide Dendrimers}

It is a dendron-like molecular construct based upon a polylysine skeleton. Lysine with its alkyl amino side-chain serves as a good monomer for the introduction of numerous of branching points. This type of dendrimer was introduced by J. P. Tam in 1988, has predominantly found its use in biological applications, e.g. vaccine and diagnostic research.

\section{Frechet-Type Dendrimers}

It is a more recent type of dendrimer developed by Hawker and Frechet based on poly-benzyl ether hyper branched skeleton. These dendrimers usually have carboxylic acid groups as surface groups, serving as a good anchoring point for further surface functionalisation, and as polar surface groups to increase the solubility of this hydrophobic dendrimer type in polar solvents or aqueous media (Yiyun et al., 2008, Hawker et al., 1993).

\section{CHEMICOPHYSICAL PROPERTIES OF DENDRIMERS}

Dendrimers are nanoscale sized that have similar dimensions to important bio-building blocks like proteins, DNA. Multiple numbers of terminal surface groups (Z) enables bio-conjugation of drugs, signalling groups, targeting moieties or biocompatibility groups. The dendrimer surfaces may be designed with functional groups to augment or resist trans-cellular, epithelial or vascular biopermeability. The interior void space may be used to encapsulate small molecule drugs, metals or imaging moieties. Encapsulating in that void space reduces the drug toxicity and facilitates controlled release. Positive biocompatibility patterns that are associated with lower generation anionic or neutral polar terminal surface groups as compared to higher generation neutral apolar and cationic surface groups. Non- or lowimmunogenicity associated with most dendrimer surfaces modified with small functional groups or polyethylene glycol (PEG). Surface groups can be modified to optimize biodistribution; receptor mediated targeting, therapy dosage or controlled release of drug from the interior space. Dendrimers have ability to be excreted from body as a function of nanoscale diameter. Dendrimers are monodisperse macromolecules, unlike linear polymers. The classical polymerization process which results in linear polymers is usually random in nature and produces molecules of different size, whereas size and molecular mass of dendrimers can be specifically controlled during synthesis (Saktivel et al., 1998). Because of their molecular architecture, dendrimers show some significantly improved physical and chemical properties when compared to traditional linear polymers. In solution, linear chains exist as flexible coils; in contrast, dendrimers form a tightly packed ball. This has a great impact on their rheological properties. Dendrimer solution has significantly lower viscosity than linear polymers. When the molecular mass of dendrimers increases, their intrinsic viscosity goes through a maximum at the fourth generation and then begins to decline. Such behaviour is unlike that of linear polymers. For classical polymers the intrinsic viscosity increases continuously with molecular mass. The presence of many chain-ends is responsible for high solubility and miscibility and for high reactivity. Dendrimers solubility is strongly influenced by the nature of surface groups. Dendrimers terminated in hydrophilic groups are soluble in polar solvents, while dendrimers having hydrophobic end groups are soluble in nonpolar solvents. A marked difference was also observed in chemical reactivity. Dendritic polyesters was debenzylated by catalytic hydrogenolysis whereas linear polyester was unreactive. Dendrimers have some unique properties because of their globular shape and the presence of internal cavities. The most important one is the possibility to encapsulate guest molecules in the macromolecule interior (Ramaswamy et al., 2003, Sonke \& Tomalia, 2005).

\section{FACTORS AFFECTING DENDRIMER PROPERTIES}

\section{Effect of $\mathbf{p H}$}

The study of structural behaviour of PAMAM dendrimers as a function of $\mathrm{pH}$, by applying molecular dynamics show that the dendrimer has an extended conformation, based on a highly ordered structure at low $\mathrm{pH}$ 
$(\mathrm{pH}<4)$. At this $\mathrm{pH}$, the interior is getting increasingly "hollow" as the generation number increases as a result of repulsion between the positively charged amines both at the dendrimer surface and the tertiary amines in the interior. At neutral $\mathrm{pH}$, back-folding occurs which may be a consequence of hydrogen bonding between the uncharged tertiary amines in the interior and the positively charged surface amines. At higher $\mathrm{pH}(\mathrm{pH}>10)$ the dendrimer contract as the charge of the molecule becomes neutral, acquiring a more spherical (globular) structure, where the repulsive forces between the dendrimer arms and between the surface groups reaches a minimum. At this $\mathrm{pH}$, the conformation has a higher degree of back-folding as a consequence of the weak "inter-dendron" repulsive forces (Gupta et al., 2007, Wang \& Imae, 2004).

\section{Effect of Solvent}

The solvation power of any solvent to solvate the dendrimer is a very important parameter when investigating the conformational state of a dendrimer. Dendrimers of all generations generally exhibit a larger extent of back-folding with decreasing solvent quality, i.e. decreasing solvation. However, being more flexible, the low generation dendrimers show the highest tendency towards back-folding as a result of poor solvation compared to the higher generation dendrimers. NMR studies performed on PPI dendrimers concluded that a nonpolar solvent like benzene, poorly solvates the dendrimers favouring intramolecular interactions between the dendrimer segments and back-folding. But, a weakly acidic solvent like chloroform can act as a hydrogen donor for the interior amines in a basic dendrimer like PPI, leading to an extended conformation of the dendrimer because of extensive hydrogen bonding between the solvent and the dendrimer amines. Both experimental as well as theoretical studies on amino-terminated PPI and PAMAM dendrimers (polar dendrimers) show the tendency that nonpolar aprotic (poor) solvents induce higher molecular densities in the core region as a result of back-folding, whereas polar solvents solvate the dendrimer arms and induce a higher molecular density on the dendrimer surface. Backfolding of the polar surface groups may expose the more hydrophobic dendrimer parts to the surroundings leading to a decreased surface polarity of the back-folded dendrimer (Chai et al., 2001).

\section{Effect of Salt}

High ionic strength (high concentration of salts) has a strong effect on charged PPI dendrimers and favours a contracted conformation of dendrimers, with a high degree of back-folding somewhat similar to what is observed upon increasing $\mathrm{pH}$ or poor solvation. At low salt conditions, the repulsive forces between the charged dendrimer segments results in an extended conformation in order to minimize charge repulsion in the structure (Gupta et al., 2007).

\section{Effect of Concentration}

In dendrimers with flexible structures the conformation is not only affected by small molecules like solvents, salts or protons, but may also be sensitive to larger objects, such as other dendrimers or surfaces which can have a great affect on the molecular density and conformation of the dendrimer. Small angle X-ray scattering (SAXS) experiments performed on PPI dendrimers (G4, G5) in a polar solvent like methanol show that the molecular conformation of dendrimers upon increasing concentration becomes increasingly contracted. This molecular contraction may minimize the repulsive forces between the dendrimer molecules and increase the ability of the dendrimers to exhibit a more tight intermolecular packing.

\section{DENDRIMER -DRUG INTERACTIONS}

Different interaction mechanisms have been explored, and they can be broadly sub-divided into three types: simple encapsulations, electrostatic interactions and covalent conjugations.

\section{Simple Encapsulation}

The ellipsoidal or spheroidal shape, empty internal cavities, and open nature of the architecture of dendrimers make it possible to directly encapsulate guest molecules into the macromolecule interior. These empty internal cavities are hydrophobic in nature, which make it suitable to interact with poorly soluble drugs through hydrophobic interactions. Moreover, the nitrogen or oxygen atoms in the internal cavities can interact with the drug molecules by hydrogen bond formation. In view of these specific properties, the relationship between the internal cavities of dendrimers and drug molecules may involve these supramolecular interactions like physical encapsulation, hydrophobic interaction or hydrogen bonding (Brownlie et al., 2004, Liu et al., 1990).

\section{Electrostatic Interaction}

The high density of functional groups like amine groups and carboxyl groups on the surface of dendrimers have potential applications in enhancing the solubility of hydrophobic drugs by electrostatic interaction. The G3 PAMAM dendrimer with an ammonia core is taken as an example. It has a much higher amino group density when compared with classical linear polymers. Non-steroidal antiinflammatory drugs with carboxyl groups, including ibuprofen, ketoprofen, diflunisal, naproxen and indomethacin, have been widely been complexed with dendrimers by electrostatic interactions. Some anticancer and antibacterial drugs have also been reported to be incorporated by this kind of interaction. The common property of these drug molecules is that they are weakly acidic drugs with carboxyl groups in the molecules (Neerman et al., 2004). 


\section{Covalent Conjugation}

The presence of large numbers of functional groups on the surface of dendrimers makes them suitable for the covalent conjugation of numerous drugs with relevant functional groups.

In this case, the drug is covalently bound to dendrimers and its release occurs via chemical or enzymatic cleavage of hydrolytically labile bonds. The encapsulation of drug molecules within hydrophobic cavities or absorption of drugs to the surface of dendrimers via electrostatic interactions preserves the chemical integrity and pharmacological properties of drug molecules, while covalent attachment of drugs to the surface groups of dendrimers through chemical bonds affords better control over drug release, facilitating the tissue targeting and controlled drug delivery.

\section{MECHANISM OF DRUG DELIVERY THROUGH DENDRIMERS}

Due to the well defined 3D structure and many surface functional groups, drug molecules can be loaded both in the interior of the dendrimers as well as attached to the surface groups as discussed earlier. Dendrimers can function as drug carriers either by encapsulating drugs within the dendritic structure or by interacting with drugs at their terminal functional groups via electrostatic or covalent bonds forming prodrug.

There are broadly two mechanisms for drug delivery:

I. First one is by in vivo degradation of drug dendrimer covalent bonding which depends on presence of suitable enzymes or an environment capable of cleaving the bonds.

II. The second one is by releasing the drug due to changes in physical environment such as $\mathrm{pH}$, temperature. This approach is independent of the external factors and takes place in cavities of the core (endo-receptor) or outer shell of receptor (exo-receptor) (Tomalia et al., 1985, Hawker \& Frechet, 1990, Hawker et al., 1993).

\section{PHARMACEUTICAL APPLICATIONS}

\section{Dendrimers in pulmonary drug delivery}

Dendrimers have been reported for pulmonary drug delivery of Enoxaparin. G2 and G3 generation positively charged PAMAM dendrimers were reported to increase the relative bioavailability of Enoxaparin by $40 \%$. The positively charged dendrimer forms complex with enoxaparin, which was effective in deep vein thrombosis after pulmonary administration (Bai et al., 2007).

\section{Dendrimer in transdermal drug delivery}

Dendrimers has been found to improve solubility and plasma circulation time via transdermal formulations and to deliver drugs efficiently. PAMAM dendrimer complex with NSAIDs (e.g. Ketoprofen, Diflunisal) have been reperted to improve the drug permeation through the skin as penetration enhancers. Ketoprofen and Diflunisal were conjugated with G5 PAMAM dendrimer and showed 3.4 and 3.2 times higher permeation. Enhanced bioavailability of PAMAM dendrimers by using indomethacin as the model drug in transdermal drug application was reported to be effective (Cheng, 2008, Chauhan \& Jain, 2003, Jevprasesphant et al., 2003).

\section{Dendrimer in oral drug delivery}

Oral drug delivery studies using the human colon adenocarcinoma cell line, Caco2, have indicated that lowgeneration PAMAM dendrimers cross cell membranes, presumably through a combination of two processes, i.e. paracellular transport and adsorptive endocytosis. Remarkably, the P-glycoprotein efflux transporter does not appear to affect dendrimers, therefore drug dendrimer complexes are able to bypass the efflux transporter. PAMAM dendrimers conjugated with the folic acid and fluorescein isothiocyanate for targeting the tumor cells and imaging respectively. DNAassembled dendrimer conjugates may allow the combination of different drugs with different targeting and imaging agents (Barbara \& Maria, 2001, Rajeshbabu et al., 2010).

\section{Dendrimer hydrogel for ocular drug delivery}

Dendrimers are especially ideal for synthesizing hydrogels, cross-linked networks that increase in volume in aqueous solution and are more similar to living tissue than any other synthetic compound. By adding polyethylene glycol or PEG groups to the dendrimers, these hydrogels have applications including cartilage tissue production and for sealing ophthalmic injuries. By synthesizing a hydrogel composed of PEGylated dendrimers that contain ocular drug molecules attached to the dendrimers efficiently deliver the drugs to the eye (Yang \& Kao, 2006).

\section{Dendrimers for controlled release drug delivery}

The anticancer drugs adriamycin and methotrexate were encapsulated into PAMAM dendrimers (i.e. $\mathrm{G}=3$ and 4) which had been modified with PEG monomethyl ether chains (i.e. 550 and $2000 \mathrm{Da}$ respectively) attached to their surfaces. A similar construct involving PEG chains and PAMAM dendrimers was used to deliver the anticancer drug 5 fluorouracil. Encapsulation of 5-fluorouracil into G4 increases in the cytotoxicity and permeation of dendrimers. The earlier discussed dendrimer drug interaction techniques are used to control the drug delivery. A third-generation dendritic unimolecular micelle with indomethacin entrapped as model 
drug gives slow and sustained in vitro release, as compared to cellulose membrane control. Controlled release of the Flurbiprofen could be achieved by formation of complex with amine terminated generation 4 (G4) PAMAM Dendrimers (Chen et al., 2004, Malik et al, 2012, Liu et al., 2000, Liu et al., 1999).

\section{Dendrimers in targeted drug delivery}

Dendrimers have ideal properties which are brought in application in targeted drug delivery system. One of the most effective cell-specific targeting agents delivered by dendrimers is folic acid PAMAM dendrimers modified with carboxymethyl PEG5000 surface chains possessed reasonable drug loading, a reduced release rate and reduced haemolytic toxicity compared with the non-PEGylated dendrimer (Kolhe et al., 2003, Mohammad \& Antony, 2006, Hawker, 2006). The star polymer were reported to give the most promising results regarding cytotoxicity and systemic circulatory half-life $(72 \mathrm{~h})$. In addition to improving drug properties such as solubility and plasma circulation time polymeric carriers can also facilitate the passive targeting of drugs to solid tumours. Combined these factors lead to the selective accumulation of macromolecules in tumour tissue, a phenomenon termed the 'Enhanced Permeation and Retention' (EPR) effect. Therefore, the anticancer drug doxorubicin was reported to be covalently bound to this carrier via an acid-labile hydrazone linkage. The cytotoxicity of doxorubicin was significantly reduced (80-98\%), and the drug was successfully taken up by several cancer cell lines. (Medina \& Mohamed, 2009, Bharali \& Khalil, 2009, Sonke \& Tomalia, 2005).

\section{Dendrimers as Nano-Drugs}

Poly(lysine) dendrimers modified with sulfonated naphthyl groups have been found to be useful as antiviral drugs against the herpes simplex virus, can potentially prevent/reduce transmission of HIV and other sexually transmitted diseases (STDs). This dendrimer-based nano-drug inhibited early stage virus/cell adsorption and later stage viral replication by interfering with reverse transcriptase and/or integrase enzyme activities. PPI dendrimers with tertiary alkyl ammonium groups attached to the surface have been shown to be potent antibacterial biocides against Gram positive and Gram negative bacteria. Poly (lysine) dendrimers with mannosyl surface groups are effective inhibitors of the adhesion of E. coli to horse blood cells in a haemagglutination assay. Chitosan-dendrimer hybrids have also been found to be useful as antibacterial agents (Sonke \& Tomalia, 2005).

\section{Dendrimers in Gene Transfection}

Dendrimers can act as vectors in gene therapy. PAMAM dendrimers have been tested as genetic material carriers. Amino-terminated PAMAM or PPI dendrimers have been reported as non-viral gene transfer agents, enhancing the transfection of DNA by endocytosis. A transfection reagent called SuperFect ${ }^{\mathrm{TM}}$ consisting of activated dendrimers is commercially available. Activated dendrimers can carry a larger amount of genetic material than viruses. SuperFectDNA complexes are characterized by high stability and provide more efficient transport of DNA into the nucleus than liposomes. The high transfection efficiency of dendrimers may not only be due to their well defined shape but may also be caused by the low $\mathrm{pK}$ of the amines (3.9 and 6.9). The low $\mathrm{p} K$ permit the dendrimer to buffer the $\mathrm{pH}$ change in the endosomal Compartment. PAMAM dendrimers functionalized with cyclodextrin showed luciferase gene expression about 100 times higher than for unfunctionalized PAMAM or for non-covalent mixtures of PAMAM and cyclodextrin. It should be noted that dendrimers of high structural flexibility and partially degraded high-generation or hyperbranched dendrimers appear to be better suited for certain gene delivery operations than intact high-generation symmetrical dendrimers (Barbara \& Maria, 2001, Christine et al., 2005, Zinselmeyer et al., 2002).

\section{TOXICITY AND PEGYLATION}

It is known that the dendrimers may have toxicity mainly attributed to the interaction of the cationic dendrimers surface with negative biological load membranes damaging cellular membranes causing hemolytic toxicity and cytotoxicity. Therefore, PAMAM dendrimers are more cationic than anionic cytotoxic. An example of interaction with lipid bilayers of cells occurs with the cationic dendrimer-G7 PAMAM which comes to form holes $15-40 \mathrm{~nm}$ in diameter, which disturbs the flow of electrolyte causing cell death.

Many toxic effects of dendrimers are attenuated at their surfaces with hydrophilic molecules and poly (ethylene glycol) (PEG) which masks the surface charge cationic dendrimers improving biocompatibility and increasing the solubility of the polymers. The pegylated dendrimers have lower cytotoxicity and longer stay in the blood than non-pegylated dendrimers. PEGylation increases the physical dendrimers size which reduces renal clearance (Buchleir et al., 1978, Uchegbu et al., 2004, Bhadra et al., 2005).

\section{CONCLUSION}

Dendrimer drug delivery systems are getting huge interest as an advantageous solution for delivering bioactive like drugs and gene. They provide a platform for the attachment of drugs or genes and their release through several mechanisms. Various applications of dendrimers have been explored during last three decades. Development in controlled polymerization and synthesis techniques have led to the emergence of well-controlled dendrimers structures with a large number of surface groups that can be utilized to display a range of biological molecules including peptides, proteins, sugars and targeting agents. The high loading capacity of 
dendrimers renders them highly attractive as carriers for delivery of chemotherapeutic agents. PEGylated and nonPEGylated dendrimers proved to encapsulate hydrophobic drug molecules into the hollow voids of their branching architecture, which enhance the aqueous solubility and stability of the encapsulated drug molecules. Both targeted and nontargeted dendrimer-drug complexes successfully penetrate across tumor's leaky vasculature and accumulate in the cancer tissue. However, targeted dendrimer-drug complexes have the added advantage of selectively binding to the receptors displayed on the surface of cancer cells, which increases their residence time on the cell surface and enhances their internalization kinetics into the cell.

\section{FUTURE PROSPECTS}

An additional area of research that is currently being explored is the development of dendrimers clusters, where several dendrimers are bound together through physical or chemical forces to assemble a multifunctional therapeutic system that incorporates the anticancer drugs, targeting ligands, and imaging agents, which will create new way for combination anticancer therapy along with in vivo imaging of the targeted tumour. Despite the effectiveness of dendrimers based drug delivery systems, their application in cancer therapies with defined dosage regimen is still not acceptable, which can be due to the difficulty of synthesizing the desired systems in large quantities at clinical grade purity for clinical trials coupled with regulatory hurdles that demand detailed characterization of the polymeric carriers along with the linkages and the incorporated drug.

\section{REFERENCES}

Bai S, Thomas C and Ahsan F. Dendrimers as a carrier for pulmonary delivery of enoxaparin, a low molecular weight heparin. J Pharm Sci, 2007; 96 (8): 2090 - 106.

Barbara K and Maria B. Dendrimers: properties and application. Acta Biochimica Polonica, 2001; 48 (1): 199-208.

Bhadra D, Bhadra S and Jain NK. PEGylated peptide based dendritic nanoparticulate system for delivery of artemether. J Drug Del Sci Tech, 2005; 15: 65-73.

Bharali DJ, Khalil M, Gurbuz M, Simone TM, Mousa SA. Nanoparticles and cancer therapy: A concise review with emphasis on dendrimers. Int J Nanomedicines, 2009; 4: 1 - 7.

Brownlie A, Uchegbu IF and Schatzlein AG. PEI-based vesicle-polymer hybrid gene delivery system with improved biocompatibility. Int J Pharm, 2004; 274, 41-52.

Buhleier E, Wehner $\mathrm{W}$ and Vogtle F. Cascade and Nonskid-chain-like Synthesis of Molecular Cavity Topologies. Synthesis, 1978; 2: 155-158.

Chai M, Niu Y, Youngs WJ and Rinaldi PL. Structure and conformation of $\mathrm{DAB}$ dendrimers in solution via multidimensional NMR techniques. J Am Chem Soc, 2001; 123: 4670- 4678 .

Chauhan AS, Jain NK. Dendrimer mediated transdermal delivery; enhanced bioavailability of Indomethacin. J. Controlled Release 2003; 96:537-540.

Chen HT, Neerman MF, Parrish AR and Simanek EE. Cytotoxicity, hemolysis, and acute in vivo toxicity of dendrimers based on melamine, candidate vehicles for drug delivery. J Am Chem Soc, 2004; 126, 10044-10048.

Cheng Y. Dendrimers as Drug carrier: applications in different routes of drug administration. J. Pharm. Sci., 2008; 97: 33-36. Christine D, Ijeoma FU and Andreas GS. Dendrimers in gene delivery. Adv. Drug Delivery Rev., 2005; 57: 2177- 2202.

Gupta U, Agashe H and Jain NK. Polypropylene imine dendrimer mediated solubility enhancement: effect of $\mathrm{Ph}$ and functional groups of hydrophobes. J Pharm Pharm Sci, 2007; 10(3): 358-67.

Hawker C and Frechet JMJ. A new convergent approach to monodisperse dendritic molecule. J. Chem. Soc. Chem. Commun., 1990; 15: 1010-1012.

Hawker C and Frechet JMJ. Preparation of polymers with controlled molecular architecture: A new convergent approach to dendritic macromolecules. J. Am. Chem. Soc., 1990; 112: 7638-7647.

Hawker C. Dendrimers: Novel polymeric nanoarchitectures for solubility enhancement. Biomacromolecules, 2006; 7(3): 649-58.

Jevprasesphant R, Penny J, Jalal R, Attwood D, McKeown $\mathrm{NB}$ and D Emanuele A. Engineering of dendrimer surfaces to enhance transepithelial transport and reduce cytotoxicity. Pharm. Res., 2003; 20: 1543-1550.

Kolhe P, Misra E, Kannan RM, Kannan S and Lai ML. Drug complexation, in vitro release and cellular entry of dendrimers and hyperbranched polymers. Int. J. Pharm., 2003; 259: 143-160.

Liu M, Kono $\mathrm{K}$ and Frechet JMJ. Water soluble dendrimer poly (ethylene glycol) starlike conjugates as potential drug carrier. Journal of Polymer Sciences, Part A, Polymer Chemistry, 1999; 37(17): 3492-3503.

Liu M, Kono K, Frechet JMJ. Water-soluble dendritic unimolecular micelles: Their potential as drug delivery agents. J. Controlled Release, 2000; 65: 121-131.

Malik A, Chaudhury S, Garg G, Tomar A. Dendrimers: A tool for Drug Delivery. Advances in Biological Research, 2012; 6(4): 165-169.

Malik N, Wiwattanapatapee R, Klopsch R, Lorenz K, Frey H, Weener JW, Meijer EW, Paulus W and Duncan R. Dendrimers: relationship between structure and biocompatibility in vitro, and preliminary studies on the biodistribution of I-125-labelled polyamidoamine dendrimers in vivo. J. Controlled Release, 2000; 65: $133-148$.

Medina SH, Mohamed EHES, Dendrimers as carriers for delivery of chemotherapeutic agents. Chemical Reviews, 2009; 109:31413157.

Mohammad N and Antony D. Crossing cellular barriers using dendrimer nanotechnologies. Current Opinion in Pharmacology, 2006; 6: 522-527.

Neerman MF, Zhang W, Parrish AR and Simanek EE. In vitro and in vivo evaluation of a melamine dendrimer as a vehicle for drug delivery. Int. J. Pharm., 2004; 281: 129-132.

Newkome GR, Yao ZQ, Baker GR and Gupta VK. Cascade molecules: A new approach to micelles. J. Org. Chem., 1985; 50(11): 2003-2006.

Pushkar S, Philip A, Pathak K and Pathak D. Dendrimers: Nanotechnology Derived Novel Polymers in Drug Delivery. Indian J. Pharm. Educ. Res., 2006; 40 (3): 153-158.

Rajesh babu V, Mallikarjun V, Nikhat SR, Srikanth G. Dendrimers: A new carrier system for Drug Delivery. International Journal of Pharmaceutical and Applied Sciences, 2010; 1(1):1-10.

Ramaswamy C, Saktivel T, Wilderspin AF, Florence $T$. Dendriplexes and their characterization. Int. J. Pharm., 2003; 254: 17-21.

Sakthivel $\mathrm{T}$ and Florence AT. Adsorption of Amphipathic Dendrons on Polystyrene Nanoparticles. Int. J. Pharm., 2003; 254: 23-26.

Saktivel T, Toth I, Florence AT. Synthesis and physicochemical properties of lipophillic polyamide dendrimers. Pharm. res., 1998; 15: 776-782.

ISonke $\mathrm{S}$ and Tomalia DA. Dendrimers in biomedical applications reflections on the Field. Advanced Drug Delivery Reviews, 2005; 57: 2106-2129. 
Tomalia DA, Baker H, Dewald J, Hall M, Kallos G, Martin S, Roeck J, Ryder J and Smith P. A New Class of Polymers: Starburst-Dendritic Macromolecules. Polym. J., 1985; 17(1): 117-132.

Uchegbu IF, Sadiq L, Pardakhty A, El-Hammadi M, Gray AI, Tetley L, Wang W, Zinselmeyer BH and Schatzlein AG. Gene transfer with three amphiphilic glycol chitosans - the degree of polymerisation is the main controller of transfection efficiency. J. Drug Target., 2004; 12: 527-539.

Wang DJ and Imae T. Fluorescence emission from Dendrimer \& its pH dependence. J. Am. Chem. Soc., 2004; 126 (41): 13204-13205.

Yang H, Kao WJ. Dendrimers for pharmaceutical and biomedical application. Journal of Biomaterial Sciences, polymer edition, 2006, 17:3-19.
Yiyun $\mathrm{C}$, Zhenhua $\mathrm{X}$, Minglu $\mathrm{M}$ and Tonguen $\mathrm{X}$ Dendrimers as Drug Carriers: Applications in Different Routes of Drug. J. Pharma. Sci., 2008; 97(1): 123-143.

Zimmerman SC, Lawless LJ. Supramolecular chemistry of Dendrimers. Topics in Current Chemistry, 2001; 217: 95-119.

Zinselmeyer BH, Mackay SP, Schatzlein AG and Uchegbu IF. The lower-generation polypropylenimine dendrimers are effective gene-transfer agents. Pharm. Res., 2002; 19: 960-967.

\section{How to cite this article:}

Surendra Tripathy, Malay K Das., Dendrimers and their Applications as Novel Drug Delivery Carriers. J App Pharm Sci. 2013; 3 (09): 142-149. 